\title{
Chloroplast DNA Copy Number Changes during Plant Development in Organelle DNA Polymerase Mutants
}

\author{
Stewart A. Morley and Brent L. Nielsen * \\ Department of Microbiology and Molecular Biology, Brigham Young University, Provo, UT, USA
}

Chloroplast genome copy number is very high in leaf tissue, with upwards of 10,000 or more copies of the chloroplast DNA (ctDNA) per leaf cell. This is often promoted as a major advantage for engineering the plastid genome, as it provides high gene copy number and thus is expected to result in high expression of foreign proteins from integrated genes. However, it is also known that ctDNA copy number and ctDNA integrity decrease as cells age. Quantitative PCR (qPCR) allows measurement of organelle DNA levels relative to a nuclear gene target. We have used this approach to determine

OPEN ACCESS

Edited by:

Sagadevan G. Mundree, Queensland University of Technology, Australia

Reviewed by: Gong-Yin Ye,

Zhejiang University, China Shashi Kumar, International Centre for Genetic Engineering and Biotechnology, India

*Correspondence:

Brent L. Nielsen brentnielsen@byu.edu

Specialty section: This article was submitted to

Plant Biotechnology, a section of the journal

Frontiers in Plant Science

Received: 15 October 2015

Accepted: 14 January 2016

Published: 04 February 2016

Citation:

Morley SA and Nielsen BL (2016)

Chloroplast DNA Copy Number Changes during Plant Development in Organelle DNA Polymerase Mutants.

Front. Plant Sci. 7:57.

doi: 10.3389/fp/s.2016.00057 changes in copy number of ctDNA relative to the nuclear genome at different ages of Arabidopsis plant growth and in organellar DNA polymerase mutants. The mutant plant lines have T-DNA insertions in genes encoding the two organelle localized DNA polymerases (PollA and PollB). Each of these mutant lines exhibits some delay in plant growth and development as compared to wild-type plants, with the PollB plants having a more pronounced delay. Both mutant lines develop to maturity and produce viable seeds. Mutants for both proteins were observed to have a reduction in ctDNA and mtDNA copy number relative to wild type plants at all time points as measured by qPCR. Both DNA polymerase mutants had a fairly similar decrease in ctDNA copy number, while the PollB mutant had a greater effect of reduction in mtDNA levels. However, despite similar decreases in genome copy number, RT-PCR analysis of PollA mutants show that PollB expression remains unchanged, suggesting that PollA may not be essential to plant survival. Furthermore, genotypic analysis of plants from heterozygous parents display a strong pressure to maintain two functioning copies of PollB. These results indicate that the two DNA polymerases are both important in ctDNA replication, and they are not fully redundant to each other, suggesting each has a specific function in plant organelles.

Keywords: chloroplast DNA, DNA polymerase mutants, genome copy number, photosynthesis, quantitative PCR

\section{INTRODUCTION}

Through the process of endosymbiosis, ancient bacteria were engulfed by precursors of eukaryotic cells, and over time most of the genes required for organelle function from these ancestral bacteria have been moved into the nucleus. This raises the question, if most genes have migrated to the nucleus, why not all of them? How do chloroplasts benefit from maintaining their genomes? Most 
evidence suggests that the unique physiological environment of chloroplasts is required for proper regulation of chloroplastspecific genes. In a recent paper, John Allen (2015) proposes, supported by significant evidence from the literature, that redox regulation of gene expression is required within the membranebound compartment. A chloroplast sensor kinase may detect disruptions in the photosynthetic electron transport chain, which responds to changes in redox conditions to activate or repress chloroplast gene expression, allowing response and regulation of photosynthesis to changing environmental conditions (Allen, 2015). Light has been shown to affect the amount of chloroplast DNA (ctDNA) during plant development (Shaver et al., 2008). Evidence for regulation of chloroplast DNA (ctDNA) by the redox state of cells has been reported in Chlamydomonas reinhardtii (Kabeya and Miyagishima, 2013), and similarly for yeast mitochondrial DNA (mtDNA; Hori et al., 2009).

Despite the importance of these organelles, chloroplast and mitochondrial genomes possess relatively few of the genes required for their functions in photosynthesis and respiration. In Arabidopsis thaliana chloroplasts there are 87 proteincoding genes and 41 rRNA and tRNA genes (Sato et al., 1999). These numbers are very similar in chloroplast genomes from other higher plant species (Palmer, 1985). The organelle genomes require fully functional transcriptional and translational machinery for expression of the genes. However, plant organelles do not use nuclear DNA replication proteins. Instead, they utilize their own unique set of nuclear-encoded organellar localized DNA replication proteins to maintain their genomes. Many of these are dual-localized to chloroplasts and mitochondria (Christensen et al., 2005; Gualberto et al., 2013; Cupp and Nielsen, 2014; Moriyama and Sato, 2014).

In this paper we focus on chloroplast genome replication and maintenance. CtDNA in higher plants has been shown to replicate by a double-displacement loop mechanism from two specific replication origins (Kolodner and Tewari, 1975; Kunnimalaiyaan and Nielsen, 1997a,b) but may also replicate by a recombination-dependent (RDR) mechanism (Oldenburg and Bendich, 2004; Rowan et al., 2010; Nielsen et al., 2010). The use of two distinct replication mechanisms has been observed for many bacterial virus genomes (Kreuzer and Brister, 2010), where one mechanism is used during the initial stage of infection and another [RDR or rolling circle (RC) replication] for rapid replication of the phage genome for incorporation into new phage particles. The use of two or more mechanisms has been discussed as a possibility for ctDNA replication in plants (Nielsen et al., 2010). Replication via a double-displacement mechanism from specific origins may be involved in maintaining low levels of the chloroplast genome in mature or quiescent cells, while recombination-dependent replication may drive rapid replication to generate high copy numbers of the genome during early stages of plant development.

Tobacco (Ono et al., 2007) and Arabidopsis (Christensen et al., 2005; Parent et al., 2011) have been found to encode two closely related bacterial-like DNA polymerases, which have been designated PolIA and PolIB. Both are dual-localized to chloroplasts and mitochondria in these species (Christensen et al., 2005). PolIB has been shown to play a role in ctDNA repair
(Mori et al., 2005; Parent et al., 2011) and mtDNA maintenance, photosynthesis, and respiration (Cupp and Nielsen, 2013). However, in rice (Kimura et al., 2002) and maize (Udy et al., 2012) a single chloroplast-localized DNA polymerase has been identified. By analysis of mutants the maize enzyme, encoded by the $w 2$ gene, appears to be the only DNA polymerase that functions in chloroplasts and may also function in mitochondria (Udy et al., 2012). There is a paralog of this gene in maize, but the protein has not been detected in chloroplasts. Both maize proteins appear to be involved in mtDNA replication (Udy et al., 2012).

Although the identification and biochemical analysis of plant organelle-localized DNA polymerases has been progressing, limited research has been reported on the role and degree of redundancy of the two DNA polymerases that are found in Arabidopsis and some other species. We have examined the effects of mutations in the A. thaliana organellar DNA polymerases on ctDNA replication by quantitative PCR (qPCR) analysis of organelle DNA levels. We provide an analysis of the effects of T-DNA insertion mutations in either of the DNA polymerase genes on plant growth and development and chloroplast genome copy numbers.

\section{MATERIALS AND METHODS}

\section{Planting and Growing Conditions}

We obtained the following T-DNA insertion lines from the Arabidopsis Biological Resource Center (Figure 1; ABRC; www.arabidopsis.org): Salk_022624 for PolIA (At1g50840); Salk_134274 (this is the same line designated polIb-1 in Cupp and Nielsen, 2013) for PolIB (At3g20540). Pots with the approximate dimensions $3 \times 3 \times 4$ (width $\times$ length $\times$ height) inches were firmly packed with potting soil and placed in a tray. The soil was then saturated with nutrient water prepared with watersoluble fertilizer (Peter's Houseplant Food). Arabidopsis seeds were planted directly onto the surface of the soil and placed in a $4^{\circ} \mathrm{C}$ cold room in the dark for up to 3 days. Plants were then moved to a growth room maintained at $22^{\circ} \mathrm{C}$ with an average surface-light exposure of $80-100 \mu \mathrm{mol} \mathrm{m} \mathrm{m}^{-2} \mathrm{~s}^{-1}$. During the first 5 days of germination trays were covered with transparent plastic covers to maintain humidity and prevent drying, after which the covers were removed.

\section{Tissue Harvesting and DNA Extraction}

Leaf tissue was harvested from plants at 7, 10, 14, and 21 dpi (days post-imbibition). Genomic DNA from these plants was then isolated following a cetyltrimethylammonium bromide (CTAB) method for isolating high quality DNA (Minas et al., 2011).

\section{Screening of T-DNA Insertion Lines}

To determine if the T-DNA insertion was present, T-DNA specific primers were used in conjunction with native gene primers. Primers were designed so that native gene primers produced a PCR product about $1 \mathrm{~kb}$ in length, and that the T-DNA insertion primer paired with the native gene primer produced a PCR product $\sim 500 \mathrm{~b}$ in length. Details of the primers used in zygosity screening are shown in Supplementary Table 1. 


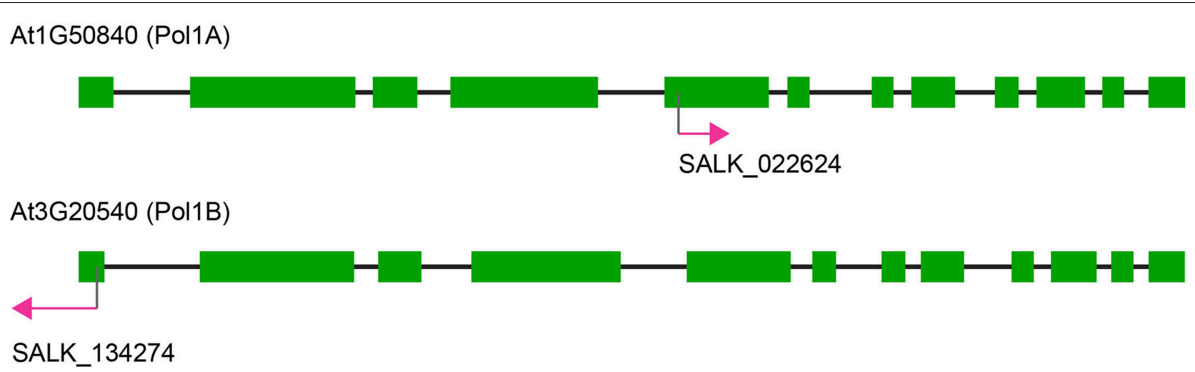

FIGURE 1 | Map of the DNA polymerase genes and T-DNA insertions. Note the overall similarity between both genes for Pol1A and Pol1B. Both genes possess 12 exons although SALK_022624 inserts in the fifth exon of Pol1A whereas SALK_134274 inserts in the first exon of Pol1B.

In order to obtain plants that were heterozygous for PollA and PolIB genes, homozygous PolIA and PolIB plants were emasculated and then pollinated from either homozygous PolIA or PolIB flowers. This cross generated offspring that were heterozygous for both PolIA and PolIB, confirmed via PCR. Seeds from the first generation of heterozygous plants were collected to screen for all possible combinations of PolIA and PolIB using PCR as described above.

\section{Genome Copy Number Analysis}

Mitochondrial and chloroplast genome copy number was analyzed using an Applied Biosystems StepOne Plus qPCR machine and PowerUp SYBR green reagents. To analyze genome copy number, sequences unique to either ctDNA or mtDNA were identified. For ctDNA analysis, the targets psbK, petD, and ndhH were used. For mtDNA analysis, these targets included nad9, orf25, and cox1. The housekeeping gene AtRpoTp was used as a positive nuclear control and a reference for $\Delta \Delta \mathrm{Ct}$ calculations. A summary of these targets and their specific genes are listed in Supplementary Table 2. Technical and biological replicates were compiled and analyzed using the $\Delta \Delta \mathrm{Ct}$ method (Schmittgen and Livak, 2008; Cupp and Nielsen, 2013).

\section{Analysis of Gene Expression Analysis in PollA Insertion Line}

mRNA was isolated from 7 dpi plants using PureLink Plant RNA Reagent (Life Technologies). RNase free DNaseI was added to remove residual DNA. Purity of mRNA was confirmed by running a small amount on a gel and checking for the absence of large DNA bands. cDNA for RT-PCR was generated from the purified mRNA using SuperScript III reverse transcriptase (Thermo Fisher). Primers for RT-PCR were designed to amplify a portion of the gene near the $3^{\prime}$ end of the mRNA. Primers for RT-PCR are described in Supplementary Table 3.

\section{Photosynthesis Assays}

Seeds from each mutant were germinated in plastic scintillation vials and grown under the same conditions as described above. At 14 dpi the vials were placed in a Licor 6400-22 Lighted Conifer Chamber Package connected to a Licor Li-6400XT analyzer. This system has the ability to measure photosynthetic rates and can automatically generate $\mathrm{CO}_{2}$ and light response curves. For this study, net photosynthetic rates of PolIA and PolIB mutants were calculated by measuring total leaf surface area. Total leaf area was calculated by scanning each plant and using ImageJ to trace and calculate surface area.

\section{RESULTS}

\section{Phenotype and Expression Analysis of Organelle DNA Polymerase Mutants}

The T-DNA insertion in PolIA is in the fifth exon of the gene, while the insertion in PolIB is in the first exon (Figure 1). The homozygous single mutant plants exhibited slight growth delays but both grow to maturity and produce seeds. Mutants in PolIB mutant plants exhibit a slower growth rate than the PolIA mutants. This pattern is consistent over time and reproducible (Figure 2; Supplementary Movie 1). This indicates that neither DNA polymerase is completely essential for development.

We previously showed that both DNA polymerases are expressed in most plant tissues during development, but there is a difference when comparing expression levels of the two genes. DNA PolIA is most highly expressed (relative to DNA PolIB) in rosette leaves, while DNA PolIB is expressed more abundantly in non-photosynthetic tissue (Cupp and Nielsen, 2013). We previously reported that in PollB mutant plants, when expression of PolIB is knocked down a substantial increase (60$70 \%)$ in PolIA expression was observed by qRT-PCR analysis (Cupp and Nielsen, 2013). We were interested to determine if a similar compensatory effect occurs for the PolIA mutant. However, relative expression of PolIB in PolIA mutant plants was not significantly different from wild-type levels (Figure 3). This suggests an important role for DNA PolIA in chloroplasts and ctDNA maintenance, while PolIB may play a more significant role in mtDNA replication and maintenance.

Our findings are consistent with expression of the Arabidopsis DNA PolIA gene compiled from microarray analysis in the Arabidopsis eFP browser (http://bar.utoronto.ca/ dev/eplant/). PolIA expression is highest in rosette leaves of wild-type plants, especially the youngest leaves, but is also high in imbibed seeds and developing flowers, and remains relatively high in cauline and older leaves. Expression of PolIA is low in embryos and siliques and in pollen (Figure 4), and is stimulated by drought and greatly repressed by osmotic stress (Nakabayashi et al., 2005; 


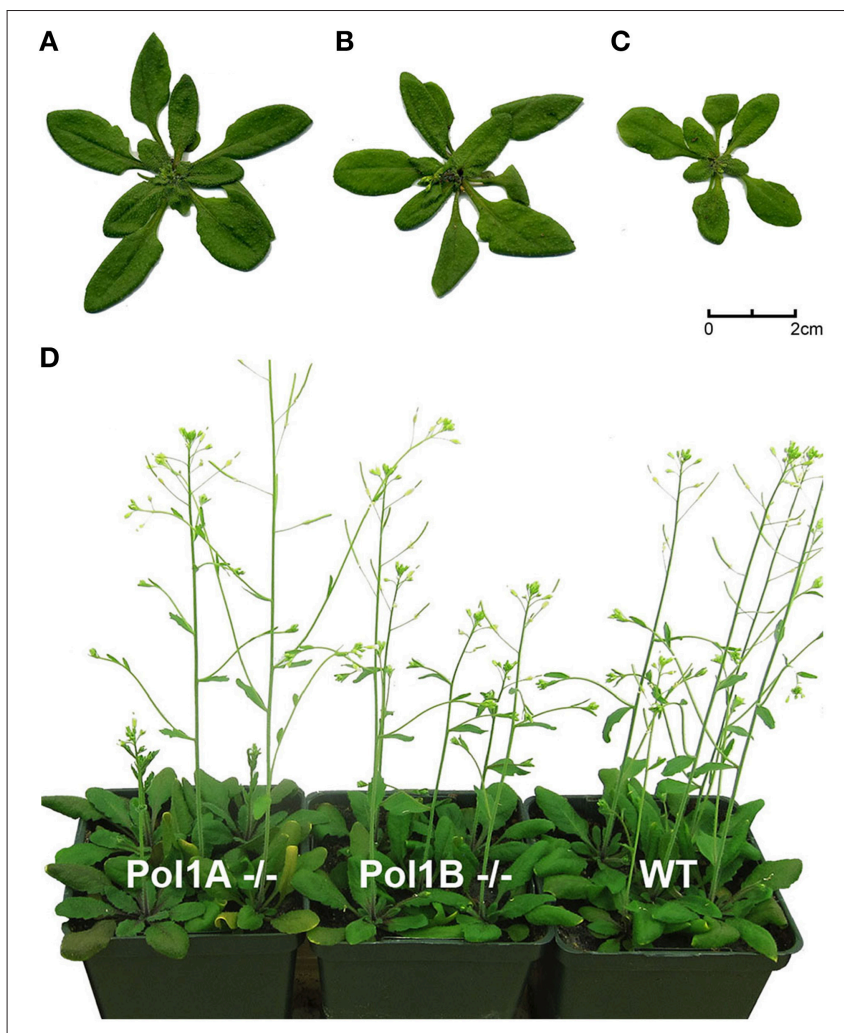

FIGURE 2 | Side by side comparison of 23 dpi WT (A), Pol1 $A^{-/-}$(B), and Pol1B $^{-/-}$(C) plants. Note the slightly delayed growth of Pol1B ${ }^{-/-}$plants and the lack of a distinguishable phenotype between WT and Pol1 $A^{-/-}$plants (D).

Schmid et al., 2005). Coexpression data (ATTED-II) indicates that the PolIA gene is coexpressed along with chloroplastlocalized RecA, OSB2 (a single-stranded DNA binding protein, Gualberto et al., 2013) and some helicase genes. These proteins may all be involved in ctDNA replication, which would be compatible with the involvement of DNA recombination in chloroplast genome replication (RDR) and/or repair. There is very little information available for DNA PolIB in these databases.

Field-inversion gel electrophoresis (FIGE) and restriction pattern analysis of ctDNA from the mutants showed no discernable differences in the mutants compared to wildtype plants (data not shown). We used a PCR assay to detect any differences in rearrangement frequency in the mitochondrial genome, as has been observed for mutants affected in mtDNA recombination (Xu et al., 2011). However, the PolIA and PolIB mutants showed no differences in rearrangement frequency, indicating that there is no major disruption or change in the mechanism for DNA replication/recombination in the individual gene mutants for ctDNA or mtDNA (not shown).

\section{CtDNA and mtDNA Copy Number Determination}

qPCR analysis of ctDNA and mtDNA levels in each of the DNA polymerase mutant lines compared to wild-type showed that

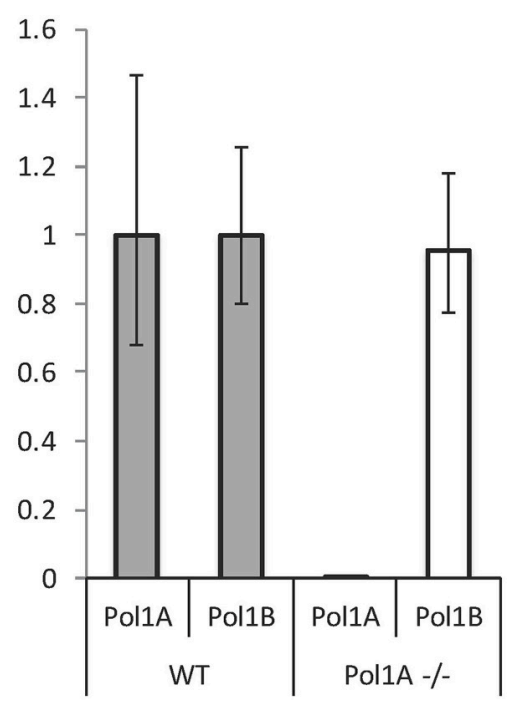

FIGURE 3 | RT-PCR of Pol1A and Pol1B expression in PollA mutant plants. Although previous work has suggested that mutation in Pol1B causes an increase in Pol1A expression, mutation of Pol1A does not affect expression of Pol1B. This experiment shows relative levels of each polymerase transcript normalized against Actin mRNA. Although mutation in Pol1A knocks down its expression, no significant change in Pol1B expression can be observed.

relative ctDNA levels and mtDNA levels, compared to the nuclear genome, are reduced in both PolIA and PolIB mutants, similar to what has been reported before for single time points (Parent et al., 2011; Cupp and Nielsen, 2013). To determine DNA levels at additional stages of growth, we analyzed samples at different time points. We examined DNA levels at 7, 10, 14, and 21 days of growth. At all time points there is a decrease in organelle DNA copy number in both mutants compared to wild-type plants of the same age for all 3 separate targets for each organelle genome at each age (Figure 5). Both PolIA and PolIB mutants showed a $\sim 30 \%$ reduction in ctDNA at 7 days, a $\sim 40 \%$ reduction at 10 and 14 days, and a 50\% reduction at 21 days. At 21 days, there is a slightly greater reduction in the PolIB mutant $(\sim 60 \%$ decrease) compared to the PolIA mutant ( $\sim 50 \%$ decrease). These results indicate that both DNA polymerases affect ctDNA copy number, in contrast with the finding in maize that a single DNA polymerase is responsible for ctDNA replication (Udy et al., 2012).

Similar but slightly different results were observed with the two mutant lines when mtDNA targets were analyzed. At 7 days the PollA mutant showed only a slight drop in mtDNA copy number, while PolIB showed nearly a 40\% drop (Figure 5), similar to what we previously reported (Cupp and Nielsen, 2013). At 10 and 14 days the PolIA mutant had a 20-40\% drop in mtDNA copy number, while in PolIB the decrease was about $50 \%$. At 21 days, the PollA mutant had a $40 \%$ decrease in mtDNA, while the PoIB mutant showed a decrease of more than $60 \%$. These results suggest that while both DNA polymerases contribute to mtDNA copy numbers, PolIB appears to play a greater role in maintenance of the mitochondrial genome. While qPCR analysis does not directly address quality of the DNA, 


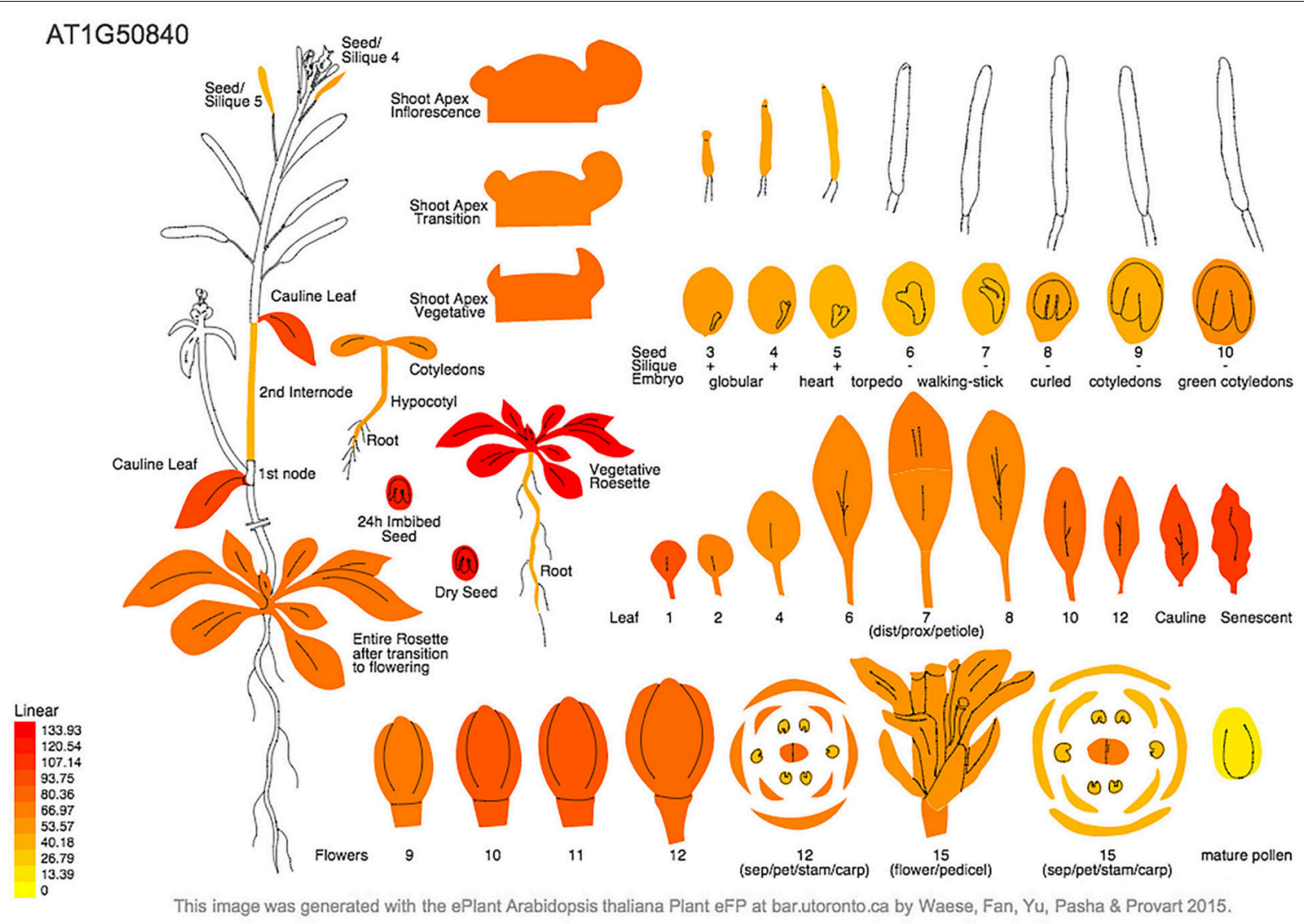

FIGURE 4 | Arabidopsis eFP browser report showing predicted PollA gene expression in different plant tissues. Expression of Pol1A is highest in rosette leaves, particularly at a young age, however, expression remains relatively high even in senescing leaves. Expression of Pol1A is lowest in seed embryos and pollen.

it does show trends over time for the mutants compared to wild-type plants, indicating changes in organelle DNA levels during development in the mutants compared to wild-type plants.

\section{Analysis of Photosynthesis in DNA Polymerase Mutants}

The decreases in organelle DNA copy number in the mutants raises a question as to whether these changes affect photosynthesis. In previous work with PolIB mutants increases in photosynthesis and related parameters were observed (Cupp and Nielsen, 2013). Current measurements showed an increase in net photosynthesis was observed in 14 dpi PolIA $^{-1-}$ plants (Figure 6). However, we acknowledge that despite careful controls during experimentation, the observed data for Poll $\mathrm{A}^{-/-}$plants may not be completely accurate. Despite this difficulty in making highly precise measurements, the data suggests that there is an increase in photosynthesis in Poll $\mathrm{A}^{-/-}$plants, although it cannot be accurately quantified at this time.

\section{Analysis of PollA x PollB Partial Double Mutants}

The results of $\mathrm{qPCR}$ analysis and previous genotyping experiments led us to believe that certain genotypes would be more beneficial to plant survival than others. To test this theory, we planted seeds on soil in the same manner described above and genotyped all plants that were able to successfully germinate and grow. As expected, none of the surviving plants were homozygous for T-DNA insertions in both DNA polymerase genes as this most likely is lethal to the plant (Figure 7). We also noticed that survival for plants possessing only one functioning DNA polymerase gene was poor. Interestingly we observed strong pressure to maintain both copies of PolIB with at least one functioning copy of PolIA. The pressure to maintain both copies of PolIB suggests higher levels of this polymerase are required to maintain healthy plants.

\section{DISCUSSION}

Analysis of mutations in the genes encoding the organellar DNA polymerases can provide helpful information for understanding their role in chloroplast DNA replication and genome maintenance. However, at the current time analysis of organelle DNA polymerase mutants has apparently only been done for Arabidopsis (Parent et al., 2011; Cupp and Nielsen, 2013) and maize (Udy et al., 2012). In maize it was shown that a single nuclear-encoded chloroplast-localized DNA polymerase (encoded by the $w 2$ gene) is responsible for nearly all ctDNA 

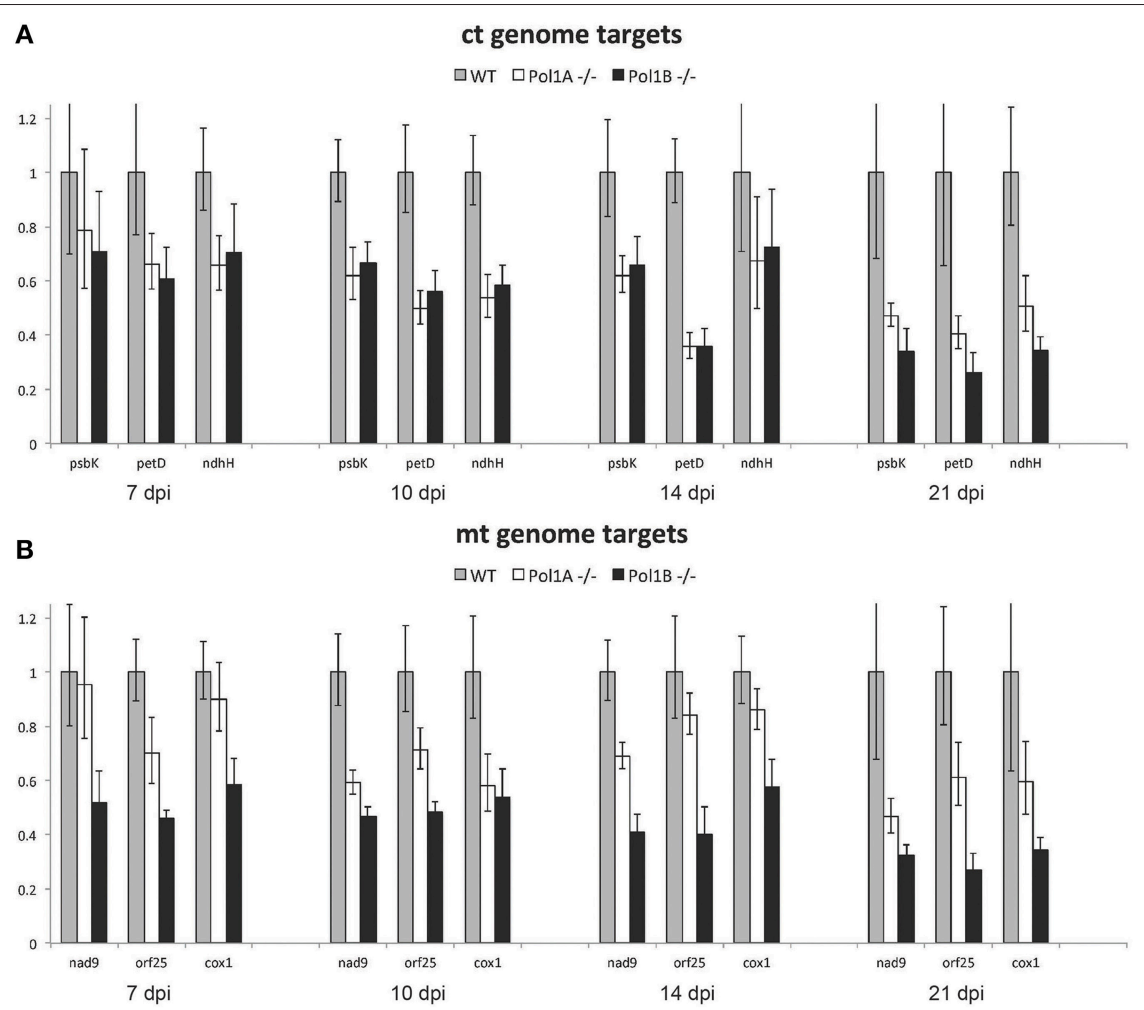

FIGURE 5 | Change in relative chloroplast and mitochondrial genome copy number. Note that mutations in Pol1A and Pol1B affect chloroplast genome copy number equally (A) however mutation of Pol1B causes a more severe drop in mitochondrial genome copy number (B). In both mutants, genome copy number gradually decreases but remains lower than wild type as the plants age.

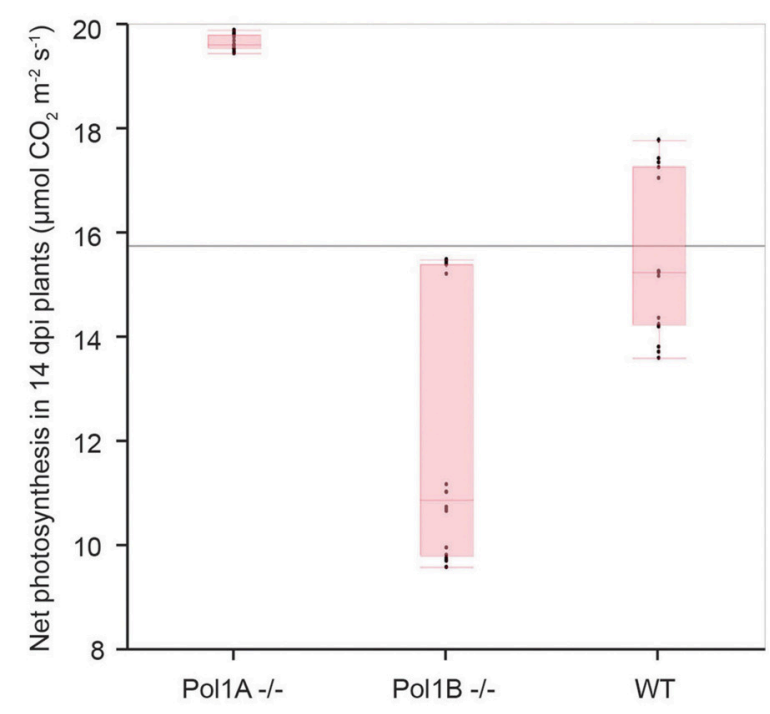

FIGURE 6 | Net photosynthetic rates in mutant plants. Observed photosynthesis rates appear to increase in Pol1 $\mathrm{A}^{-/-}$mutants.

replication (Udy et al., 2012). In contrast, our results show that both PolIA and PolIB are required to maintain normal growth of A. thaliana (Figure 2, Supplementary Movie 1, Cupp and Nielsen, 2013).
Both of the previous reports on Arabidopsis focused on PolIB, which indicated effects on mtDNA copy number and mitochondrial structure (Cupp and Nielsen, 2013) and on plastid DNA repair (Parent et al., 2011). In this paper, we have focused on PolIA, and show that it plays a role along with PolIB in ctDNA replication as measured by copy number analysis. This analysis also indicates that PolIA contributes to a lesser extent in mtDNA maintenance. Mutants in each DNA polymerase gene have a limited effect on phenotype, with PolIB plants growing the slowest, while PolIA plants grow only slightly slower than wild type plants.

Analysis of partial double mutants indicates a strong preference for at least one copy of the PolIB gene. As expected, no viable homozygous double mutants were observed, indicating that at least one copy of one of the DNA polymerases is required for growth, although growth is progressively affected by the loss of either the second PolIA or PolIB allele. As mentioned previously, there is a strong pressure to maintain at least two functioning copies of either DNA polymerase gene, and an even stronger pressure to maintain both PolIB genes with at least one functioning PolIA gene. This suggests that PolIB is much more essential to plant survival and may also be needed at higher expression levels to support a healthy plant. This is in line with our previous report that PolIB mutants are haploinsufficient while PolIA is not, which suggests an additive effect of functional PolIB gene copy number (Cupp and Nielsen, 2013). 

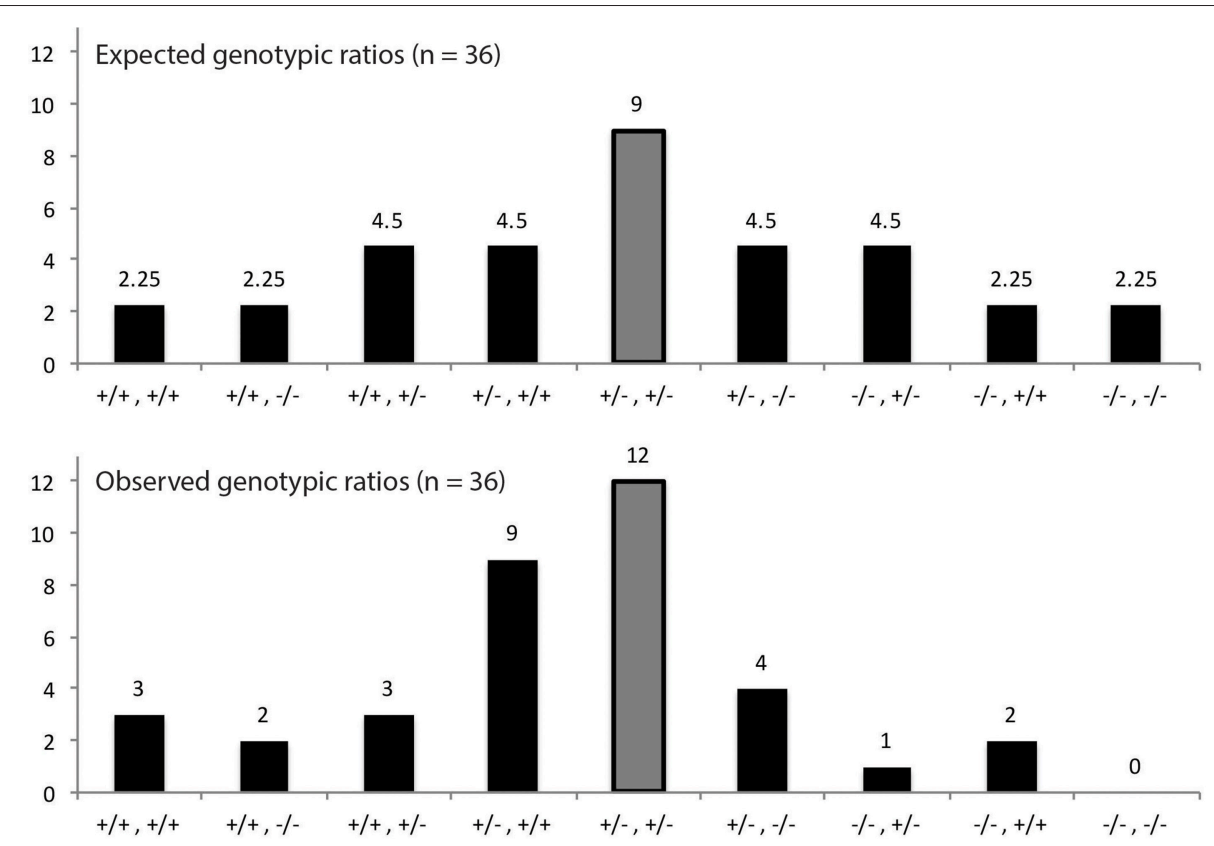

FIGURE 7 | Proportion of genotypes from DNA PollA x PollB crosses. The results come from 36 plants that were able to successfully grow on soil. The horizontal axes represent the possible genotype combinations starting with PollA and followed by PollB (e.g., $+/-,+/-$ represents PollA $\mathrm{A}^{+/-}$, PollB ${ }^{+/-}$, respectively). The middle bar represents the heterozygous combination of genes and is highlighted gray for convenience. Because the results are only from surviving plants, certain genotypes were not observed, such as PollA ${ }^{-/-}$, PollB ${ }^{-/-}$as this combination most likely is lethal to the plant. A particularly interesting genotype was PollA ${ }^{+/-}$, PollB ${ }^{+/+}$which was present in an uncharacteristically high number of plants.

TABLE 1 | Prediction of PollA and PollB organelle localization.

\begin{tabular}{lllll}
\hline Prediction program & \multicolumn{2}{c}{ PollA $^{*}$} & \multicolumn{2}{c}{ PollB $^{*}$} \\
\hline & Ct & Mt & Ct & Mt \\
TargetP & 0.928 & 0.314 & 0.588 & 0.741 \\
PCLR & 0.995 & - & 0.915 & - \\
Predotar & 0.950 & 0.100 & 0.600 & 0.450 \\
\hline
\end{tabular}

*Each prediction program returns the likelihood of each resulting protein localizing to either chloroplasts (Ct) or mitochondria (Mt). This prediction is made based on the amino acid sequence of each polymerase.

Expression of the DNA polymerase genes appears to be very high in young developing tissues, especially in meristems (Kimura et al., 2002). PollA is expressed most abundantly in developing and rosette leaves (Figure 4; Cupp and Nielsen, 2013), which agrees with the data available from online expression databases. In contrast, PolIB is expressed highly (relative to PolIA) in non-photosynthetic tissues (Cupp and Nielsen, 2013). However, both are expressed in all tissues. The higher expression of PolIA in leaves suggests that it may play an important role in ctDNA replication. However, the small effect of a homozygous insertion mutant for this gene on plant growth indicates that the PolIB gene can at least partially complement the PolIA mutation.

A significant increase in PolIA expression was observed in homozygous mutant PolIB plants (Cupp and Nielsen, 2013). In contrast, in homozygous PolIA mutants there is no significant change in PollB gene expression (Figure 3). PollA homozygous mutants show an increase in net photosynthesis (Figure 6). Photosynthesis was also affected in PolIB mutants (Cupp and Nielsen, 2013). There may be an inverse relationship between mtDNA levels and net photosynthesis. It may be a decrease in mtDNA, which would affect mitochondrial function, causes a compensatory increase in chloroplast function, including photosynthesis. Thus, while mutants in both genes share some similarities (reduction in growth rate and organelle genome copy numbers and effect on photosynthesis), there are differences in the levels of these effects that strongly suggest different functions for the two DNA polymerases.

Although, both DNA polymerases have been shown to be dual targeted to chloroplasts and mitochondria, we hypothesize that chloroplasts rely more on Pol1A whereas mitochondria rely more on Pol1B for DNA replication. We hypothesize that a mutation in Pol1B causes increased expression of Pol1A to make up for the loss of function of PollB proteins. In the reverse scenario, mutation of PollA has a less severe effect, and Pol1B may compensate for loss of function of PolIA without the need for higher PolIB expression. Further supporting this hypothesis are localization predictions based on protein sequence analysis. When the protein sequences for PolIA and PolIB are analyzed by localization prediction programs Target P (Emanuelsson et al., 2007), PCLR, (Schein et al., 2001) and Predotar (https://urgi.versailles.inra.fr/predotar/ predotar.html), PolIA is consistently predicted to localize to chloroplasts more strongly than mitochondria while PolIB is 


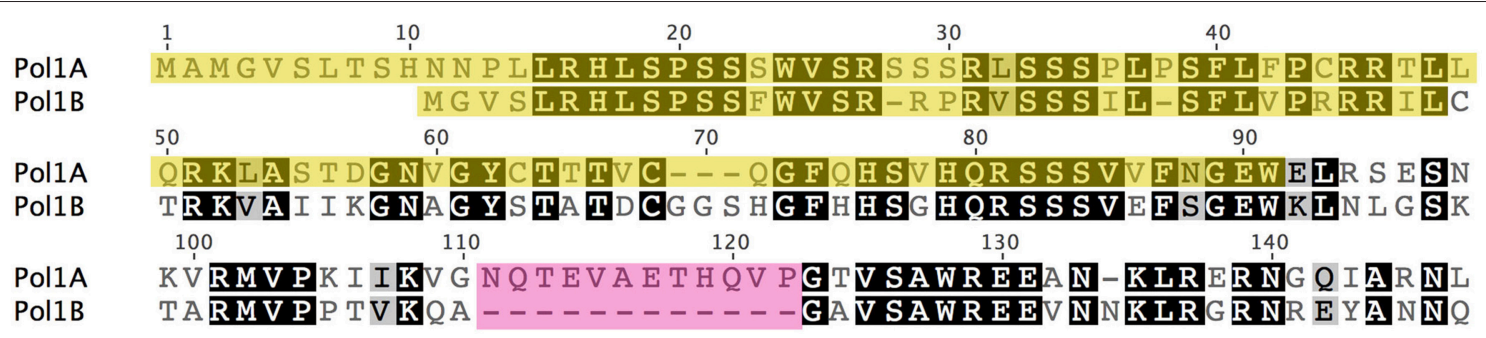

FIGURE 8 | Predicted signal peptides of PollA and PollB and sequence homology in the early region of each polymerase protein. Predictions of each protein's signal peptide was made using ChloroP. Residues highlighted in yellow represent the predicted signal peptide to be cleaved after localization. Note that despite a much shorter predicted signal peptide, PollB continues to maintain high homology with PollA for $\sim 60$ more residues. The first region of dissimilarity between the two polymerases is highlighted in pink.

most strongly predicted to localize to mitochondria. A summary of these results can be found in Table 1. A more detailed analysis using ChloroP (Emanuelsson et al., 1999) predicts that the first 91 residues of PolIA whereas only the first 36 of PolIB serve as a signal peptide for PolIB, which may help explain the differences in preferred localization. However, PolIB maintains high homology with PolIA beyond its predicted signal for $\sim 60$ residues (Figure 8). Thus, while the genes and protein products are highly homologous, they have some significant differences at the N-terminal and other internal regions, contributing to the observation that the two DNA polymerases are not fully redundant to each other.

In contrast to the computer predictions, both PollA and PolIB have been shown to be dual-targeted to chloroplasts and mitochondria (Christensen et al., 2005). However, the two DNA polymerases may not be equally localized to both organelles at all stages of plant development. It was reported that plastid localization of PolIA was only obtained when the entire $5^{\prime}$ UTR was included in the GFP fusion construct. When the UTR was deleted, initiation of protein synthesis occurred only at the annotated start codon and localization became dualtargeted. The 5'UTR lacks an in-frame upstream start codon, suggesting that an alternate non-AUG start codon was used (Christensen et al., 2005). Localization may vary depending on growth conditions, which could dictate which form of the protein is translated and thus which organelle it is targeted to. This may also play a role in the localization of the proteins when one of the DNA polymerase genes is knocked out in the TDNA insertion lines. The absence of one DNA polymerase may trigger signal(s) for expression of a form of the other DNA polymerase that can compensate for the mutated enzyme. This could explain some of the slight differences in growth rate and other characteristics between the two mutants. The proposed presence of an alternate mechanism for ctDNA replication could also explain why disruption of one or both of the mapped origins (ori) is not lethal, while some of the linear fragments generated still map near the mapped ori regions (Mühlbauer et al., 2002; Scharff and Koop, 2006). The confirmation and characterization of different replication mechanisms and differential localization of the organellar DNA polymerases during plant development or in response to mutation or stresses deserves further study.
It is interesting that of the four species for which organellar DNA polymerase genes have been characterized, Arabidopsis and tobacco, which are dicotyledonous plants, have two organelle localized DNA polymerases that both appear to be essential for normal growth and replication of chloroplast and mitochondrial genomes. In contrast, maize and rice, which are monocots, appear to have a single DNA polymerase that is responsible for substantially all ctDNA replication. Analysis of organelle DNA polymerases in additional species will be required to determine whether this is a consistent pattern, which would suggest significant differences in the replication machinery for plants from these two lineages.

Chloroplast genome copy numbers per cell are highest in young photosynthetically active leaves. Chloroplast genome copy number varies widely between tissues, ranging from 3 to 275 copies per plastid in leaf cells of different developmental stages (Zoschke et al., 2007; Liere and Borner, 2013). For other species there are 10-400 copies of the chloroplast genome per plastid, translating to 1000-50,000 genome copies per plant cell (also see Boffey and Leech, 1982; Tymms et al., 1983). This number has been given as a compelling basis for chloroplast genetic engineering. Such high copy numbers could theoretically lead to high expression of introduced genes. Indeed, high yields of gene products in engineered chloroplasts have been reported (Grevich and Daniell, 2005; Maliga and Bock, 2011).

\section{CONCLUSION}

In summary, there are two closely related organelle-localized DNA polymerases in A. thaliana. While mutants in either gene have only a slight effect on plant growth and net photosynthesis, the two enzymes do not appear to be fully redundant. Mutation of Pol1B causes a more drastic effect on growth compared to the effect of mutation in Pol1A. This is supported by genome copy number analysis. Mutation of either DNA polymerase causes a similar decrease in ctDNA copy number, while mutation of Pol1B causes a more substantial reduction in mtDNA genome copy number than PollA mutation. While knockdown of PolIB resulted in increased expression of PolIA, suggesting compensation for the loss of PolIB (Cupp and Nielsen, 2013), knockdown of PolIA did not lead to any significant change in PolIB expression (this work). However, PolIA mutants exhibit a 
small increase in net photosynthesis, suggesting some adjustment in plants to the reduction in organelle DNA levels. Analysis of double mutants suggests that while homozygous mutants of either DNA polymerase are still viable, there is a strong pressure to maintain two functioning copies of PolIB or at the least two functioning copies of either DNA polymerase. These findings indicate that both are important for plant organelle genome replication and plant development, and suggest distinct roles for PolIA and PolIB in Arabidopsis. A better understanding of the dynamics and controls of ctDNA copy numbers are important to improve chloroplast genetic engineering to overexpress introduced genes, which is relevant to this special topic issue.

\section{FUNDING}

This research was supported in part by a Brigham Young University Mentoring Environment Grant and by the National Institutes of Health (USA) (R15GM066787).

\section{AUTHOR CONTRIBUTIONS}

SM and BN designed the experiments and wrote the manuscript. SM performed most of the experiments, analyzed data, prepared figures, and edited the manuscript. $\mathrm{BN}$ obtained funding, directed the experiments, performed some of the computational gene analysis, and wrote the manuscript.

\section{REFERENCES}

Allen, J. F. (2015). Why chloroplasts and mitochondria retain their own genomes and genetic systems: colocation for redox regulation of gene expression. Proc. Natl. Acad. Sci. U.S.A. 112, 10231-10238. doi: 10.1073/pnas. 1500012112

Boffey, S. A., and Leech, R. M. (1982). Chloroplast DNA levels and the control of chloroplast division in light-grown wheat leaves. Plant Physiol. 69, 1387-1391. doi: 10.1104/pp.69.6.1387

Christensen, A. C., Lyznik, A., Mohammed, S., Elowsky, C. G., Elo, A., Yule, R., et al. (2005). Dual-domain, dual-targeting organellar protein presequences in Arabidopsis can use non-AUG start codons. Plant Cell 17, 2805-2816. doi: 10.1105/tpc.105.035287

Cupp, J. D., and Nielsen, B. L. (2013). A shift in cellular dynamics occurs in an Arabidopsis organellar DNA polymerase IB mutant. Physiol. Plant. 149, 91-103. doi: 10.1111/ppl.12009

Cupp, J. D., and Nielsen, B. L. (2014). Minireview: DNA replication in plant mitochondria. Mitochondrion 19, 231-237. doi: 10.1016/j.mito.2014. 03.008

Emanuelsson, O., Brunak, S., Von Heijne, G., and Nielsen, H. (2007). Locating proteins in the cell using TargetP, SignalP and related tools. Nat. Protoc. 2, 953-971. doi: 10.1038/nprot.2007.131

Emanuelsson, O., Nielsen, H., and Von Heijne, G. (1999). ChloroP, a neural network-based method for predicting chloroplast transit peptides and their cleavage sites. Protein Sci. 8, 978-984. doi: 10.1110/ps.8.5.978

Grevich, J. J., and Daniell, H. (2005). Chloroplast genetic engineering: recent advances and future perspectives. Critical Rev. Plant Sci. 24, 83-107. doi: $10.1080 / 07352680590935387$

Gualberto, J. M., Mileshina, D., Wallet, C., Niazi, A. K., Weber-Lotfi, F., and Dietrich, A. (2013). The plant mitochondrial genome: dynamics and maintenance. Biochimie 100, 107-120. doi: 10.1016/j.biochi.2013.09.016

Hori, A., Yoshida, M., Shibata, T., and Ling, F. (2009). Reactive oxygen species regulate DNA copy number in isolated yeast mitochondria by triggering

\section{ACKNOWLEDGMENTS}

We appreciate advice from Drs. Sally Mackenzie and Vikas Shedge, University of Nebraska, Lincoln. Tyler Augenstein and Hannah Yokum assisted with various aspects of growing plants, harvesting DNA, performing PCR screens, and assisting with photosynthesis measurements. Dr. Dennis Eggett of the BYU Statistics Department assisted in data analysis and error calculations.

\section{SUPPLEMENTARY MATERIAL}

The Supplementary Material for this article can be found online at: http://journal.frontiersin.org/article/10.3389/fpls.2016. 00057

\begin{abstract}
Supplementary Movie 1 | Time lapse of WT, Pol1A $-/-$, and Pol1B-/plants growing side by side. In this video, plants can be seen growing at slightly different rates based on their mutation. The plants in the first frame are $7 \mathrm{dpi}$ (days post-imbibition). Black frames represent times when lights were turned off to simulate the night cycle. In total, the plants can be seen growing from $7 \mathrm{dpi}$ to $32 \mathrm{dpi}$. In this video, no difference is noted between WT and Pol1 $\mathrm{A}^{-/-}$ plants. However, Pol1 $\mathrm{B}^{-/-}$plants can be seen lagging slightly behind WT and $\mathrm{Pol}_{1} \mathrm{~A}^{-/-}$. This delay is most pronounced when plants begin to put up shoots.
\end{abstract}

Supplementary Table 1 | Primers used for qPCR analysis of mitochondrial and chloroplast genome copy number.

Supplementary Table 2 | Primers used for RT-PCR analysis of PollA and PollB expression.

Supplementary Table 3 | Primers used for zygosity testing.

recombination-mediated replication. Nucl. Acids Res. 37, 749-761. doi: 10.1093/nar/gkn993

Kabeya, Y., and Miyagishima, S. (2013). Chloroplast DNA replication is regulated by the redox state independently of chloroplast division in Chlamydomonas reinhardtii. Plant Physiol. 161, 2102-2112. doi: 10.1104/pp.113.216291

Kimura, S., Uchiyama, Y., Kasai, N., Namekawa, S., Saotome, A., Ueda, T., et al. (2002). A novel DNA polymerase homologous to Escherichia coli DNA polymerase I from a higher plant, rice (Oryza sativa L.). Nucl. Acids Res. 30, 1585-1592. doi: 10.1093/nar/30.7.1585

Kolodner, R. D., and Tewari, K. K. (1975). Chloroplast DNA from higher plants replicates by both the Cairns and rolling circle mechanism. Nature 256, 708-711. doi: 10.1038/256708a0

Kreuzer, K. N., and Brister, J. R. (2010). Initiation of bacteriophage T4 DNA replication and replication fork dynamics: a review in the Virology Journal series on bacteriophage T4 and its relatives. Virol. J. 7:358. doi: 10.1186/1743422X-7-358

Kunnimalaiyaan, M., and Nielsen, B. L. (1997a). Chloroplast DNA replication: mechanism, enzymes and replication origins. J. Plant Biochem. Biotechnol. 6, 1-7. doi: 10.1007/BF03263000

Kunnimalaiyaan, M., and Nielsen, B. L. (1997b). Fine mapping of replication origins (oriA and oriB) in Nicotiana tabacum chloroplast DNA. Nucl. Acids Res. 25, 3681-3686. doi: 10.1093/nar/25.18.3681

Liere, K., and Borner, T. (2013). "Development-dependent changes in the amount and structural organization of plastid DNA" in Plastid Development in Leaves During Growth and Senescence, eds B. Biswal, K. Krupinska, and U. C. Biswal (Dordrecht: Springer), 215-237. doi: 10.1007/978-94-007-5724-0_11

Maliga, P., and Bock, R. (2011). Plastid biotechnology: food, fuel, and medicine for the $21^{\text {st }}$ century. Plant Physiol. 155, 1501-1510. doi: 10.1104/pp.110.170969

Minas, K., Mcewan, N. R., Newbold, C. J., and Scott, K. P. (2011). Optimization of a high-throughput CTAB-based protocol for the extraction of qPCR-grade DNA from rumen fluid, plant and bacterial pure cultures. FEMS Microbiol. Lett. 325, 162-169. doi: 10.1111/j.1574-6968.2011.02424.x 
Mori, Y., Kimura, S., Saotome, A., Kasai, N., Sakaguchi, N., Uchiyama, Y., et al. (2005). Plastid DNA polymerases from higher plants, Arabidopsis thaliana. Biochem. Biophys. Res. Commun. 334, 43-50. doi: 10.1016/j.bbrc.2005.06.052

Moriyama, T., and Sato, N. (2014). Enzymes involved in organellar DNA replication in photosynthetic eukaryotes. Front. Plant Sci. 5:480. doi: 10.3389/fpls.2014.00480

Mühlbauer, S. K., Lössl, A., Tzekova, L., Zou, Z., and Koop, H. U. (2002). Functional analysis of plastid DNA replication origins in tobacco by targeted inactivation. Plant J. 32, 175-184. doi: 10.1046/j.1365-313X.2002.01408.x

Nakabayashi, K., Okamoto, M., Koshiba, T., Kamiya, Y., and Nambara, E. (2005). Genome-wide profiling of stored mRNA in Arabidopsis thaliana seed germination: epigenetic and genetic regulation of transcription in seed. Plant $J$. 41, 697-709. doi: 10.1111/j.1365-313X.2005.02337.x

Nielsen, B. L., Cupp, J. D., and Brammer, J. (2010). Mechanisms for maintenance, replication and repair of the chloroplast genome in plants. J. Exp. Bot. 61, 2535-2537. doi: 10.1093/jxb/erq163

Oldenburg, D. J., and Bendich, A. J. (2004). Most chloroplast DNA of maize seedlings in linear molecules with defined ends and branched forms. J. Mol. Biol. 335, 953-970. doi: 10.1016/j.jmb.2003.11.020

Ono, Y., Sakai, A., Takechi, K., Takio, S., Takusagawa, M., and Takano, H. (2007). NtPolI-like1 and NtPolI-like2, bacterial DNA polymerase I homologs isolated from BY-2 cultured tobacco cells, encode DNA polymerases engaged in DNA replication in both plastids and mitochondria. Plant Cell Physiol. 48, 1679-1692. doi: 10.1093/pcp/pcm140

Palmer, J. D. (1985). Comparative organization of chloroplast genomes. Annu. Rev. Genet. 19, 325-354. doi: 10.1146/annurev.ge.19.120185.001545

Parent, J. S., Lepage, E., and Brisson, N. (2011). Divergent roles for the two PolIlike organelle DNA polymerases of Arabidopsis. Plant Physiol. 156, 254-262. doi: 10.1104/pp.111.173849

Rowan, B. A., Oldenburg, D. J., and Bendich, A. J. (2010). RecA maintains the integrity of chloroplast DNA molecules in Arabidopsis. J. Exp. Bot. 61, 2575-2588. doi: 10.1093/jxb/erq088

Sato, S., Nakamura, Y., Kaneko, T., Asamizu, E., and Tabata, S. (1999). Complete structure of the chloroplast genome of Arabidopsis thaliana. DNA Res. 6, 283-290. doi: 10.1093/dnares/6.5.283

Scharff, L. B., and Koop, H. U. (2006). Linear molecules of tobacco ptDNA end at known replication origins and additional loci. Plant Mol. Biol. 62, 611-621. doi: $10.1007 / \mathrm{s} 11103-006-9042-\mathrm{x}$
Schein, A. I., Kissinger, J. C., and Ungar, L. H. (2001). Chloroplast transit peptide prediction: a peek inside the black box. Nucleic Acids Res. 29:e82. doi: 10.1093/nar/29.16.e82

Schmid, M., Davison, T. S., Henz, S. R., Pape, U. J., Demar, M., Vingron, M., et al. (2005). A gene expression map of Arabidopsis thaliana development. Nat. Genet. 37, 501-506. doi: 10.1038/ng1543

Schmittgen, T. D., and Livak, K. J. (2008). Analyzing real-time PCR data by the comparative C(T) method. Nat. Protoc. 3, 1101-1108. doi: $10.1038 /$ nprot.2008.73

Shaver, J. M., Oldenburg, D. J., and Bendich, A. J. (2008). The structure of chloroplast DNA molecules and the effects of light on the amount of chloroplast DNA during development in Medicago truncatula. Plant Physiol. 146, 1064-1074. doi: 10.1104/pp.107.112946

Tymms, M. J., Scott, N. S., and Possingham, J. V. (1983). DNA content of Beta vulgaris chloroplasts during leaf cell expansion. Plant Physiol. 71, 785-788. doi: 10.1104/pp.71.4.785

Udy, D. B., Belcher, S., Williams-Carrier, R., Gualberto, J. M., and Barkan, A. (2012). Effects of reduced chloroplast gene copy number on chloroplast gene expression in maize. Plant Physiol. 160, 1420-1431. doi: 10.1104/pp.112.204198

Xu, Y. Z., Arrieta-Montiel, M. P., Virdi, K. S., de Paula, W. B. M., Widhalm, J. R., Basset, G. J., et al. (2011). MutS HOMOLOG1 is a nucleoid protein that alters mitochondrial and plastid properties and plant response to high light. Plant Cell 23, 3428-3441. doi: 10.1105/tpc.111.089136

Zoschke, R., Liere, K., and Börner, T. (2007). From seedling to mature plant: Arabidopsis plastidial genome copy number, RNA accumulation and transcription are differentially regulated during leaf development. Plant J. 50, 710-722. doi: 10.1111/j.1365-313X.2007.03084.x

Conflict of Interest Statement: The authors declare that the research was conducted in the absence of any commercial or financial relationships that could be construed as a potential conflict of interest.

Copyright (๑) 2016 Morley and Nielsen. This is an open-access article distributed under the terms of the Creative Commons Attribution License (CC BY). The use, distribution or reproduction in other forums is permitted, provided the original author(s) or licensor are credited and that the original publication in this journal is cited, in accordance with accepted academic practice. No use, distribution or reproduction is permitted which does not comply with these terms. 